\section{Externally Driven Nonlinear Oscillator, Painlevé Test, First Integrals and Lie Symmetries}

\section{W.-H. Steeb and N. Euler}

Department of Applied Mathematics and Nonlinear Studies, Rand Afrikaans University Auckland Park 2006,

South Africa

Z. Naturforsch. 48a, 943-944 (1993);

received May 12, 1993

For arbitrary constants $c_{1}, c_{2}$ and an arbitrary smooth functions $f$ the driven anharmonic oscillator $\mathrm{d}^{2} u / \mathrm{d} t^{2}+$ $c_{1} \mathrm{~d} u / \mathrm{d} t+c_{2} u+u^{3}=f(t)$ cannot be solved in closed form. We apply the Painleve test to obtain the constraint on the constants $c_{1}, c_{2}$ and the function $f$ for which the equation passes the test. We also give the Lie symmetry vector field and first integrals for this equation.

For arbitrary constants $c_{1}, c_{2}$ and an arbitrary smooth function $f$ the anharmonic oscillator

$$
\frac{\mathrm{d}^{2} u}{\mathrm{~d} t^{2}}+c_{1} \frac{\mathrm{d} u}{\mathrm{~d} t}+c_{2} u+u^{3}=f(t)
$$

cannot be solved in closed form.

We apply the Painleve test $[1,2,3]$ to obtain the constraint on the constants $c_{1}, c_{2}$ and the function $f$ for which (1) passes the test. The constraint on $c_{1}$ and $c_{2}$ gives an algebraic equation and the constraint of $f$ is a linear differential equation. We solve these equations and give the Lie point symmetry and the first integral for this special case of (1).

Let us first discuss the Painlevé test for (1). A remark is in order for applying the Painleve test for non-autonomous systems. The coefficients that depend on the independent variable must themselves be expanded in terms of $t-t_{1}$, where $t_{1}$ is the pole position and we use the identity $t \equiv\left(t-t_{1}\right)+t_{1}$. If nonautonomous terms enter the equation at lower order than the dominant balance the above mentioned expansion turns out to be unnecessary whereas if the nonautonomous terms are at dominant balance level they must be expanded with respect to $t-t_{1}$. We assume that $f$ does not enter the expansion at dominant level.

Reprint requests to Prof. Dr. W.-H. Steeb, Department of Applied Mathematics and Nonlinear Studies, Rand Afrikaans University, P.O. Box 524, Auckland Park 2006, South Africa. email: whs @,rau3.rau.ac.za.
Before we study (1) we give a brief review of the special case

$$
\frac{\mathrm{d}^{2} u}{\mathrm{~d} t^{2}}+c_{1} \frac{\mathrm{d} u}{\mathrm{~d} t}+c_{2} u+u^{3}=0
$$

where $c_{1}$ and $c_{2}$ are constants. Equation (2) is considered in the complex domain with $c_{1}$ and $c_{2}$ real. For the sake of simplicity we do not change the notation. Inserting the Laurent expansion

$$
u(t)=\sum_{j=0}^{\infty} a_{j}\left(t-t_{1}\right)^{j-n},
$$

where $t_{1}$ denotes the pole position, yields $n=1$ and $a_{0}^{2}=-2$. The expansion coefficients $a_{1}, a_{2}$, and $a_{3}$ are determined by

$$
\begin{aligned}
& 3 a_{1} a_{0}=c_{1}, \quad 3 a_{2} a_{0}=-c_{2}-3 a_{1}^{2}, \\
& 4 a_{3}=c_{1} a_{2}+c_{2} a_{1}+a_{1}^{3}+6 a_{0} a_{1} a_{2} .
\end{aligned}
$$

The expansion coefficient $a_{4}$ is arbitrary in expansion (3) if

$$
c_{1}^{2}\left(2 c_{1}^{2}-9 c_{2}\right)=0 \text {. }
$$

This means $r=4$ is a so-called resonance (compare $[1,2,3]$ and references therein). The solution $c_{1}=0$ is the trivial case. To summarize: If $2 c_{1}^{2}=9 c_{2}$, then the general solution of (2) can be expressed in terms of Jacobi elliptic functions. For this case (i.e. $2 c_{1}^{2}=9 c_{2}$ ) we can find an explicitly time-dependent first integral, namely

$I(t, u, \dot{u})=\exp \left(\frac{4}{3} c_{1} t\right)\left(\left(\dot{u}+\frac{c_{1} u}{3}\right)^{2}+\frac{1}{2} u^{4}\right)$.

If condition (5) is satisfied, then (2) admits two Lie symmetry vector fields

$Z_{1}=\frac{\partial}{\partial t}, \quad Z_{2}=-\frac{c_{1}}{3} \exp \left(\frac{c_{1} t}{3}\right) u \frac{\partial}{\partial u}+\exp \left(\frac{c_{1} t}{3}\right) \frac{\partial}{\partial t}$.

Let us now consider (1). Inserting the ansatz

$$
u(t)=\sum_{j=0}^{\infty} u_{j}(t) \phi(t)^{j-n}
$$

with $n=1$ into (1), we find at the resonance $r=4$ the condition

$$
-27 \sqrt{-2} \frac{\mathrm{d} f}{\mathrm{~d} t}-27 \sqrt{-2} c_{1} f-18 c_{2} c_{1}^{2}+4 c_{1}^{4}=0 .
$$


Since $\sqrt{-2}$ is imaginary and $c_{1}$ and $c_{2}$ are real, it follows that (9) decomposes into two equations, namely

$$
\frac{\mathrm{d} f}{\mathrm{~d} t}+c_{1} f=0
$$

and condition (5). The general solution of (10) is given by

$$
f(t)=C e^{-c_{1} t} .
$$

Consequently,

$$
\frac{\mathrm{d}^{2} u}{\mathrm{~d} t^{2}}+c_{1} \frac{\mathrm{d} u}{\mathrm{~d} t}+\frac{2}{9} c_{1}^{2} u+u^{3}=C \exp \left(-c_{1} t\right)
$$

passes the Painlevé test. Equation (12) admits one Lie symmetry vector field, namely $Z_{2}$ given by (7). Now

[1] W.-H. Steeb and N. Euler, Nonlinear evolution equations and Painlevé test. World Scientific Publishing, Singapore 1988.

[2] N. Euler and W.-H. Steeb, Continuous symmetries, Lie algebras and differential equations, Bibliographisches Institut, Mannheim 1992.

[3] W.-H. Steeb, Invertible point transformation and nonlinear differential equations, World Scientific Publishing, Singapore 1993.
(12) can be derived from a Lagrangian function

$$
\mathscr{L}(u, \dot{u}, t)=e^{c_{1} t}\left(\frac{1}{2} \dot{u}^{2}-V(u, t)\right),
$$

where

$$
V(u, t)=\frac{1}{9} c_{1}^{2} u^{2}+\frac{1}{4} u^{4}-C u e^{-c_{1} t} .
$$

Thus we can apply Noether's theorem to find a first integral from the Lie symmetry vector field $Z_{2}$. We obtain

$$
\begin{aligned}
& I(t, u, \dot{u}) \\
& \quad=\exp \left(\frac{4}{3} c_{1} t\right)\left(\left(\dot{u}+\frac{c_{1} u}{3}\right)^{2}+\frac{1}{2} u^{4}-2 C u e^{-c_{1} t}\right) .
\end{aligned}
$$

We used REDUCE [4] and $C++$ [5] for most of the calculations performed in this paper.

[4] W.-H. Steeb and D. Lewien, Algorithm and computations with REDUCE, Bibliographisches Institut, Mannheim 1992.

[5] W.-H. Steeb, D. Lewien, and O. Boine-Frankenheim: Object-oriented programming in science with $\mathrm{C}++$, Bibliographisches Institut, Mannheim 1993. 
Effects of Long-Range Coupling on Aggregation

\section{E. Canessa}

International Centre for Theoretical Physics, Trieste, Italy

Wei Wang

Physics Department, Nanjing University, People's Republic of China

Z. Naturforsch. 48a, 945-946 (1993); received June 21, 1993

Numerical simulations of a $2 \mathrm{D}$ biharmonic equation $\nabla^{4} u=0$ show that a transition from dense to multibranched growth is only a consequence of long-range coupling on the pattern formation of fractal aggregates.

PACS numbers: $68.70 .+\mathrm{w}, 05.40 .+\mathrm{j}, 61.50, \mathrm{Cj}$

It has been recently shown that screening, due to free charges, strongly diversifies the pattern that grow in electrostatic fields [1, 2] (see also [3]). By solving the Poisson equation, a transition from dense to multibranched growth has been found which depends on the potentials $\phi^{0}$ and $\phi^{i}$ at two boundaries, the distance between them, $L-l$, and an inverse screening length $\lambda$. In this note we illustrate an extention of this problem based on the biharmonic (BH) equation $\nabla^{4} u=0$ in $2 \mathrm{D}$ isotropic defect-free media. We prove here that the transition from dense to multibranched growth is only a consequence of long-range coupling between displacements on pattern formation, and that in the present model the transition appears when the velocity on the growing surface presents a minimum as also occurs in Poisson growth.

These new results are important because of the physical relevance of the $\mathrm{BH}$ equation, as follows from the well-known Kuramoto-Sivashinsky (KS) equation that models pattern formation in different physical context, such as chemical reaction-diffusion systems and cellular gas flames in the presence of external stability factors $[4,5]$. The $\mathrm{BH}$ equation also appears, e.g., when describing the deflection of a thin plate subjected to uniform loading over it surface with fixed edges or within the steady, slow $2 \mathrm{D}$ motion of a viscous fluid [6].

Reprint requests to canessae @ ictp.trieste.it, or Dr. E. Canessa, ICTP-International Centre for Theoretical Physics, Condensed Matter Group (MB), P.O. Box 586, I-34100 Trieste, Italy.
Herein we consider the simplest version of the KS equation, i.e. we assume static solutions. This allows us to include long-range coupling through the discretization of the $\mathrm{BH}$ equation on lattice sites involving values of $u$ at thirteen mesh points. This is the crucial difference with respect to Laplacian and Poisson models in which iterative procedures are carried out around ( four) next-nearest neighbours (nn) only.

Figure 1 shows the final stage of a $\mathrm{BH}$ pattern displaying features, in circular geometry, of a transition from dense to multibranched growth when attaching one particle at each step. Below the transition point $r_{l} \sim 0.6 L$, the fractal dimension approaches the value for Laplacian growth within error bars. To generate this $\mathrm{BH}$ fractal pattern we have set the derivative boundary condition (DBC), that is necessary along the $r$-direction, equal to zero and the growth probability $P$ proportional to $\nabla^{2} u$ (corresponding to the potential in [1]). Above $r_{l}$ this figure is a demonstration that long-range coupling is the most relevant aspect for this phenomenon alternative to screening as suggested by Louis et al.

For planar growth we use periodic boundary conditions in the $x$-direction and estimate the DBC along the $y$-direction from the analytical solution $3 u^{0} / L$ by fixing $u^{i}=0$ and (rescaled) $\left.\phi^{0} \equiv \nabla^{2} u\right|_{y=L} \approx 6 u^{0} / L^{2}$.

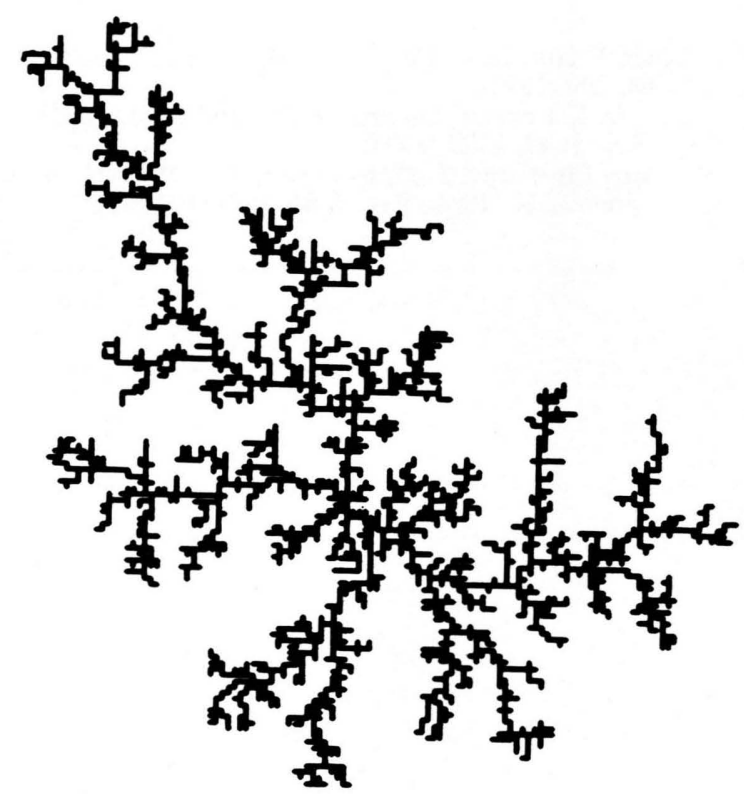

Fig. 1. Biharmonic pattern displaying the transition.

0932-0784 / 93 / 0800-0945 \$01.30/0. - Please order a reprint rather than making your own copy. 


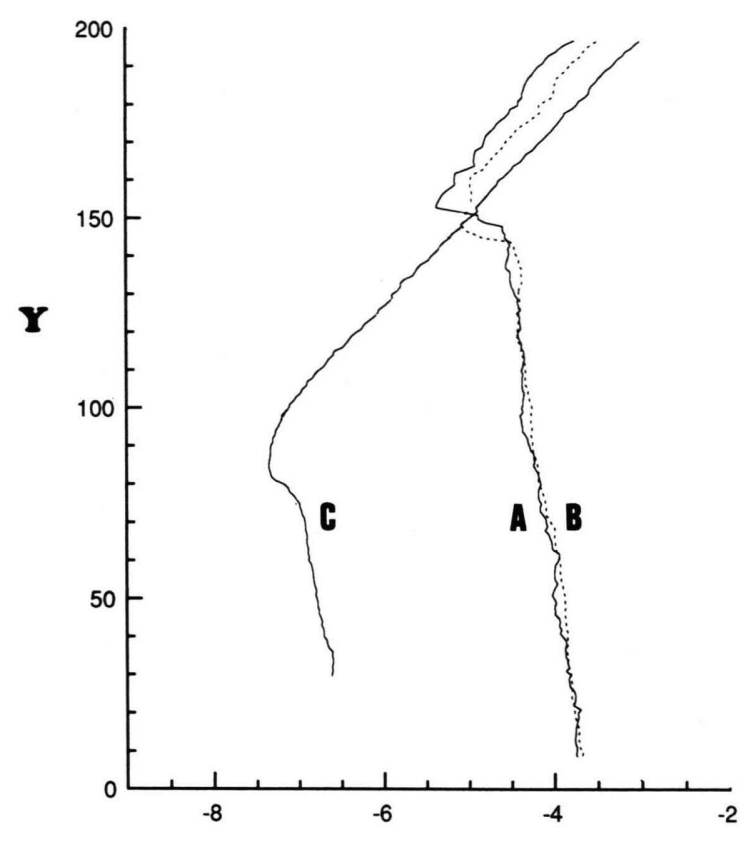

\section{LOG (V)}

Fig. 2. Growth velocity $v$ along the $y$-direction proportional to $\left|\nabla^{2} u\right|$ (A) or displacement $|u|$ (B). (C) is for Poisson growth [1].

[1] E. Louis, F. Guinea, O. Pla, and L. M. Sander, Phys. Rev. Lett. 68, 209 (1992).

[2] J. Castella, E. Louis, F. Guinea, O. Pla, and L. M. Sander, Phys. Rev. E 47, 2729 (1993).

[3] W. Wang, Phys. Rev. E 47, to appear July 1993.

[4] K. Sneppen et al., Phys. Rev. A 46, R 7351 (1992).
In Fig. 2 we plot results for the grow velocity $v$ along the $y$-direction, which we relate to the averaged value of $P$, e.g., equal to $\nabla^{2} u$ (curve A), to include nn sites, or to the displacement $u$ (curve B). In curve C, $v$ is set proportional to the field $\left|\phi_{i, j}-\phi^{i}\right|$ following [1]. In this geometry we find that the transition appears when $v$ on the growing surfaces presents a minimum, as also occurs in Poisson growth (PG: curve C). However, the BH patterns in the dense region (not shown) are not that denser as in $[1,2]$. The reason for this is that for PG the transition occurs at much smaller $v$ than for BH growth, hence an Eden-like pattern can be generated. Above this transition multibranched fractals appear in both models, but for $\mathrm{BH}$ growth the transition line depends on the system size. It is remarkable that the three curves in Fig. 2 present parallel slopes above and below their respective transition points.

The transition obtained by numerically solving the BH part of the KS equation might not be necessarily similar to the Hecker transition. But the KS equation can be transformed to look somewhat like the NavierStokes equation for a potential flow with negative viscosity which may be somehow related to the recent analysis in [7] concerning electrochemical deposition.

[5] H. and B. Ma, Phys. Rev. E 47, 3738 (1993).

[6] J. R. Melrose, D. B. Hibbert, and R. C. Ball, Phys. Rev. Lett. 65, 3009 (1990).

[7] V. Fleury, J.-N. Chazalviel, and M. Rosso, Phys. Rev. Lett. 68, 2942 (1992).

Nachdruck - auch auszugsweise - nur mit schriftlicher Genehmigung des Verlages gestattet 